\title{
La construcción social de la Costa Brava. Una aproximación bibliográfica
}

\author{
Sergi Yanes Torrado* \\ Universitat Oberta de Catalunya (España)
}

\begin{abstract}
Resumen: Tras más de medio siglo de desarrollo turístico intensivo, la Costa Brava sigue siendo una de las regiones turísticas más populares del Mediterráneo. Durante su proceso de construcción, expansión y renovación, este destino vacacional ha sido objeto de multitud de investigaciones dentro del ámbito de las ciencias sociales. Estos trabajos (en forma de estudios, artículos, monografías, pero también de novelas e incluso guías) se han preocupado de un modo u otro por reflejar y comprender las lógicas económicas, políticas y turísticas de las sucesivas transformaciones territoriales, residenciales, culturales o medioambientales que han ido definiendo la región durante esos años. Este artículo se presenta como un recorrido histórico y bibliográfico por esas mismas investigaciones. Tiene como objetivo tratar de establecer relaciones entre temáticas y disciplinas para señalar luego, los principales elementos que han intervenido en la producción de lo que hoy reconocemos bajo el nombre de Costa Brava.
\end{abstract}

Palabras Clave: Costa Brava; Ciencias sociales; Turismo; Investigaciones; Bibliografía.

The social construction of the Costa Brava. A bibliographic approach

Abstract: Over half a century of intensive tourism development, the Costa Brava remains one of the most popular tourist regions in the Mediterranean. During its process of construction, expansion and renovation, this leisure destination has been subject of a large number of social science research studies. These works (papers, articles, monographs, but also novels and even guides) have been concerned, in some way, to reflect and understand the economic, political and tourist logics of territorial, residential, cultural and environmental transformations that have been defining the region during those years. This article provides a historical and bibliographical overview of such investigations. Its main objective is to establish relations between topics and disciplines in order to point out the main ingredients that have intervened on the production of what we today recognize under the name of Costa Brava.

Keywords: Costa Brava; Social sciences; Tourism; Research; Bibliograph.

\section{Introducción}

La Costa Brava, región de la costa catalana situada entre Blanes y la frontera francesa, es uno de los destinos turísticos más importantes del Mediterráneo y uno de los más longevos de la industria del sol y playa en Europa. Desde inicios del siglo XX, pero sobre todo a partir de su segunda mitad, este tramo de costa entre los municipios catalanes de Blanes y Portbou, ha sido un popular punto de encuentro para un sinfín de visitantes. Una intensa afluencia que ha sido posible gracias a una infraestructura turística basada en establecimientos hoteleros, sistemas de transporte y una amplia oferta de ocio y diversión. El laborioso proceso de producción del destino que ha implicado ha transformado recursos de naturaleza territorial, cultural, laboral, legal y económica. Estas implicaciones han ocupado la atención de un número importante de investigadores que, desde disciplinas vinculadas de forma más o menos directa a las ciencias sociales, han elaborado una producción científica que ha ayudado a constituir un cuerpo teórico, metodológico y empírico de gran valor.

Universitat Oberta de Catalunya (España); E-mail: yanes.sergio@gmail.com; https://orcid.org/0000-0001-9699-6831 
La finalidad de este texto es realizar un repaso a este repertorio sobre la Costa Brava, principalmente el producido a partir de la segunda mitad del siglo XX. Mediante un ejercicio de vinculación de textos, trato de mostrar qué abordajes han sido recurrentes y qué ausencias son la que destacan, cuál ha sido la genealogía de esta área de estudio y qué trato ha recibido hasta hoy. Para ello, he recurrido a bibliografía académica -artículos científicos, ensayos o monografías- que analiza o describe algún aspecto constitutivo de la Costa Brava. Cabe señalar de antemano que una parte destacada de la labor de búsqueda y lecturas de referencias fue realizada entre 2012 y 2015 , durante la elaboración del marco teórico de mi tesis doctoral. He estimado conveniente ampliar y dar salida a un contenido que habitualmente ocupa un lugar marginal en el informe final, pero que sin duda contiene información útil para orientar a aquellos investigadores que quieran adentrarse en este objeto de estudio y requieran partir de los antecedentes o elaborar un estado de la cuestión. Este es precisamente el fin que se le presupone a cualquier trabajo bibliográfico, presentar y discutir sobre el objeto de estudio, presentar sus controversias y trazar posibles itinerarios de investigación. Para todo ello, se hace imprescindible una labor de reconstrucción del mismo, que sitúe reiteraciones, debilidades y/o ausencias.

Ahora bien, no se trata de una reconstrucción bibliográfica sobre un tema en concreto, sino más bien sobre un área geográfica particular que abarca varios temas. Por tanto, he tomado la decisión de no ubicarme dentro de los límites sabidos de una u otra disciplina. Como tantos otros que moldean este trabajo, este es un criterio que se vincula con la adopción de un enfoque constructivista que asume que los objetos -en este caso la Costa Brava- no son substantivos sino sociales, es decir, resultado de interpretaciones, prácticas e interacciones situadas en contextos históricos y políticos particulares. El trabajo analítico, los debates suscitados y las formas de representar el conocimiento acumulado, son parte del sistema de relaciones que conforman el objeto y sus múltiples versiones. Por esto, he estimado también oportuno incluir algunas fuentes primarias (novelas y ensayos) así como eventos (coloquios, reuniones públicas o seminarios abiertos) que considero relevantes para el conocimiento y la generación de problematizaciones y puntos de vista sobre el área. Por otro lado, he preferido no detenerme en la innumerable producción circunscrita a la historia local de cada uno de los 24 municipios que conforman la Costa Brava, y en general, a aquella vinculada con la etnología y el estudio de la cultura tradicional. Como se verá, hay algunas excepciones, ya sea por su relevancia respecto al objeto de estudio o por su papel en el desarrollo de las ciencias sociales en la Costa Brava.

En texto cierra con algunos apuntes -a modo de conclusiones- que he considerado pertinente destacar: el legado multidisciplinar dejado a lo largo de los años, el tipo de acercamiento analítico que ha predominado en cada época o el interés depositado en la promoción de debates y jornadas sobre la Costa Brava. Así mismo se incluyen unas últimas palabras que invitan a pensar las implicaciones que los resultados de este recorrido bibliográfico tienen sobre el propio objeto de estudio.

No hace falta decir que la riqueza del conocimiento vinculado a esta zona se agota con esta cronología descriptiva. Ante esto, he decidido priorizar las investigaciones centradas en aspectos constitutivos de la Costa Brava y discriminar aquellas otras que remiten a ella de forma más secundaria o circunstancial. Para aclarar un poco esta cuestión y situar la genealogía ontológica del objeto, considero necesario desarrollar primero algunas consideraciones sobre su origen mismo. Como mostraré a continuación, la Costa Brava es una entidad social y geográfica que posee algunas particularidades.

\section{La producción del paisaje turístico de la Costa Brava}

Controversias a un lado1, la autoría del nombre "Costa Brava" se adjudica al periodista Ferran Agulló, quien propone de identificar el territorio litoral que va de Blanes a Portbou bajo un topónimo común. Ahora bien, la idea original de materializar este espacio costero en una marca turística debe vincularse necesariamente también a Lluís Duran i Ventosa, abogado y reconocido dirigente de la Lliga Regionalista ${ }^{2}$. Duran fue uno de los principales diseñadores e impulsores de la arquitectura política y económica que terminaría gestante la Mancomunidad de Cataluña (1914-1925), exponente del proyecto de unidad moral y territorial del catalanismo burgués del primer cuarto de siglo. La constitución de la Mancomunidad vino precedida de otros proyectos, uno de ellos, tal vez el que más recorrido y amplitud adquirió con el paso de los años, fue este de la Costa Brava, una operación simbólica y mediática destinada a comercializar un producto turístico capaz de posicionar nuevamente en Cataluña como potencia marítima internacional (Quintana, 2004). El anhelo casi mitológico de un pueblo levantando astilleros en cada puerto y de una marina mercante todopoderosa conquistando las Américas, se traslada ahora al campo del turismo y el progreso urbano. Se trata en definitiva del proyecto de unas élites que 
basan buena parte de su dominio de clase en la urbanización del país y el desarrollando de vías de circulación de personas y capitales.

El proyecto de Duran arranca en 1908 con el apoyo de Ferran Agulló, quien como hemos avanzado acuña el nombre de Costa Brava (a espejo de la costa brava mallorquina y a inspiración de la Côte d'Azur francesa ${ }^{3}$ ) para identificar una zona hasta entonces dispersa bajo un topónimo común. Los textos que difunde Agulló juegan con los clichés del "marco incomparable" y la "tierra de contrastes", y suponen un alegato favorable al desarrollo de infraestructuras de comunicación y hoteleras para hacer entender a los habitantes de esos pueblos que su salvación "és la indústria dels forasters, de la qual viuen, no alguns pobles, sinò nacions enteres, com Suïssa" (Quintana, 2004: 54). La voz literaria que acompaña al producto turístico Costa Brava se recrea en la "sublime belleza" del lugar, en la "fuerza moral y pedagógica" de la naturaleza y en la "esencia cultural" de las gentes que habitan sus pueblos. Duran i Agulló coinciden en la necesidad de dotar a la marca Costa Brava de ciertos rasgos históricos y culturales que sean pintorescos a ojos de la modernidad urbana. En esta tarea, será bien aprovechado el trabajo del historiador Manuel Cazurro, responsable junto a Puig i Cadafalch de las excavaciones en Empúries. Cazurro publicó en 1913 la Guía ilustrada de las ruinas de Ampurias y Costa Brava Catalana, siendo también uno de los primeros en reconocer y dar uso al topónimo de nuevo cuño.

Durante este periodo se da una cierta tensión entre la modernización social impulsada por la Mancomunitat y el acercamiento romántico y positivista al litoral catalán, un territorio en vías de "descubrimiento" por las capas urbanas ilustradas. La por entonces escasa investigación social recibe con optimismo el abandono de las metodologías decimonónicas y la adopción de nuevas técnicas de investigación (Calvo, 1997), pero también se muestra incapaz ante el poder simbólico que ejercen periodistas, artistas y escritores a la hora de configurar el paisaje y dotar al territorio de una entidad ajena a sus componentes históricos, sociales y culturales. En esta tarea turistificadora, destaca el papel que ejercen durante los años 20 los escritores catalanes de tendencia primitivista, así como los artistas que una década después se asientan en poblaciones como Tossa de Mar, Cadaqués o Port de la Selva -es el caso de Josep M. de Sagarra, Francesc Gimeno i Arasa o Rafael Benet i Vancells-. La naturaleza idílica y las gentes de pueblo serán fuente de inspiración para el "noucentisme mediterrani" y algunas de las vanguardias europeas personificadas en pintores como Marc Chagall o Pablo Picasso (Francesc Roca, 2000). El melodrama de Juli Vallmitjana, A la costa brava (1923), es también un buen ejemplo de todo esto.

A pesar de las contradicciones que enseguida genera la defensa del "litoral inmaculado" -paisaje áspero e inhóspito, soporte de la vida elemental de sus pobladores- con la operación en marcha, su posicionamiento estético se va adaptando ágilmente a los recursos turísticos, y, por lo tanto, al "relato Costa Brava". Así, paulatinamente el medio natural se transforma en paisaje y el paisaje rural en paisaje turístico (Quintana, 2004). Aunque no consiga aún traspasar con éxito las barreras locales, este tramo del litoral, ya como Costa Brava, toma la forma de entidad geográfica y se hace un lugar en los mapas, guías y cartas, consiguiendo un grado de reconocimiento similar al de los topónimos históricos.

Esta unificación de los pueblos costeros no sólo es nominal. "Costa Brava" es también la verbalización de un proyecto que sitúa en segundo plano las particularidades identitarias y organizativas de la vida política, económica y administrativa de sus pueblos, y, por tanto, la capacidad de ejercer su soberanía en materia espacial. Es la imposición de una nueva forma de significar, entender y administrar el territorio y el paisaje. El S'agaró de Josep Ensesa, los proyectos -frustrados- de ciudad jardín en Blanes o de ciudad balneario en Sant Feliu de Guíxols, son algunos ejemplos.

Durante esta década y la posterior, se publican obras que responden ya a este giro turístico del territorio. Algunos ejemplos son La Costa Brava (1929) de Vicente Solé de Sojo, Petita Guia de la Costa Brava i del Baix Empordà. Palamós Sant Joan de Palamós, Calonge, Sant Antoni i la Fosca (1930) de Lluís Colomer Moret, o La Costa Brava (1932) una de las muchas guías ilustradas que publica el Centre Excursionista de Catalunya, una de las instituciones que más colabora en su promoción turística.

El avance de lo simbólico promueve y facilita el cumplimiento de la segunda columna del proyecto original, el desarrollo inmobiliario, sea hotelero o de residencia vacacional. El fervor constructivo acaba siendo tal, que en 1935 la Conselleria d'Obres Públiques de la Generalitat republicana anuncia que uno de los cometidos del recién fundado Patronato de la Costa Brava, deberá ser evitar que "el dret de la propietat privada arribi a anul-lar el gaudi públic de la Costa Brava i enlletgir el seu magnific aspecte natural". Pero aquí, la fuerza mayor de la Guerra Civil (1936-1939) también se impone y se cortocircuitan todas las iniciativas turísticas. Otro más que valioso documento que atestigua aquellos años lo encontramos en la novela de Nancy Johnstone Hotel in Spain ${ }^{5}$, escrita en Tossa de Mar durante los primeros meses de 1937. 
Pasada la posguerra humana, si inicia la posguerra económica. El proyecto de país de Lluís Durán verá la luz, aunque de una forma bastante diferente a como lo ideó medio siglo atrás. La primera piedra la pone la Ley de Suelo de 1956, que transforma por completo el marco legal y normativo del desarrollo urbano existente hasta el momento. Su cometido no es otro que el de allanar el terreno al despliegue industrial y abrir vías para el desarrollo económico capitalista. Pero esta Ley del Suelo no permite explicar por completo la hegemonía inmobiliaria que, durante los años 60 y 70, para ello debemos rescatar el Decreto 157/63 de Liberalización industrial y la Ley 197/11963 sobre Centros y Zonas de Interés Turístico Nacional, dos reglamentos que consagran el laissez faire en el planeamiento municipal. A partir de ellos y usando el marco general de la Ley de 1956, se levantan por doquier instalaciones industriales, fábricas, naves, hoteles, apartamentos, fincas, restaurantes, campings, etc. La muerte de Lluís Durán en 1954 le impidió ser testigo de todo lo que estaba por venir.

El inicio, al menos material, del boom turístico en la Costa Brava, tiene otro actor fundamental: las touroperadoras europeas, principalmente las británicas. Su robusta capacidad económica y la garantía política y legal que la dictadura franquista ofrece a sus inversiones, se conjugan para levantar una extensa planta hotelera que llevará a la resortización del territorio. La Costa Brava obtiene durante estas décadas la unidad funcional que hasta entonces no tenía. A partir de aquí, la comprensión de sus múltiples realidades estará bajo el techo de un objeto de estudio unitario y reificado.

A falta de trabajos científicos con un cierto sentido del rigor metodológico, de nuevo la literatura ofrece documentos de gran valor para aproximarnos a los rasgos sociales de aquel momento. Para tal fin, se hace imposible no mencionar los ejercicios antropológicos que Josep Pla realiza en Guía de la Costa Brava (1941) o Cadaqués (1947). Como hijo de Palafrugell, la Costa Brava y sus pueblos, sus paisajes, su arquitectura y sus gentes, son elementos evocados de manera muy recurrentes a lo largo de la densa obra de Josep $\mathrm{Pla}^{6}$. A parte de Pla, también se deben destacar otras novelas que, quizás, por ser de autores menos reconocidos, pasaron más desapercibidas en aquel momento. Algunos ejemplos son Una piragua en la Costa Brava de Paul Fidrmuc (1948), Your guide to la Costa Brava de Douglas Clyne (1959), La Costa Brava de Néstor Luján (1958), o la negra Five roads to S'Agaró (1958) de K. G. Ballard (pseudónimo de Holly Roth). Por último, y en clave local, cabe destacar también el trabajo recopilatorio de Joan Torrent titulado La Costa Brava. Vista pels escriptors catalans (1958).

\section{El interés académico por la Costa Brava. Primeros pasos.}

La publicación de Le Paysage humain de la Costa Brava (1966), la monumental tesis doctoral de la geógrafa francesa Yvette Barbaza (1966), es un punto de inflexión. La obra de Barbaza no solo es el primer acercamiento riguroso realizado desde las ciencias sociales ${ }^{7}$ a los pueblos del litoral catalán, también coloca por primera vez al turismo en un lugar central del análisis. Los límites territoriales y paisajísticos de aquel producto de márketing llamado Costa Brava, son reconocidos por primera vez como contorno de una entidad humana y cultural. La heterogeneidad de pueblos, costumbres, labores, artes, creencias, arquitecturas y modos de organizar el tiempo y el espacio, son circunscritos en el marco general de las "gentes de la Costa Brava". En su análisis exhaustivo de la geografía humana, cultural, económica y territorial, Barbaza retrata un mundo que está desapareciendo inexorablemente, y en este sentido, cumple también el papel de acta de defunción. El trabajo de Barbaza no deja indiferente y será referencia obligatoria para toda la producción científica venidera

Le Paysage humain de la Costa Brava nace también en un momento en el que la producción académica relativa a la Costa Brava es aún inexistente. Los esfuerzos van por otro lado. Estamos en el inicio del boom turístico y el orden desarrollista lo único que pide es un marco normativo laxo ${ }^{8}$, no estudios que den a conocer los rasgos antropológicos del lugar. El proyecto urbanizador es también un proyecto de destrucción que, a veces borra y a veces se vale de las particularidades del territorio con el fin de convertirlo en suelo.

Las voces discordantes con esta forma de hacer y pensar son escasas, si bien algunas también contundentes. Como ejemplo de ello, resulta interesante rescatar las palabras del sociólogo Mario Gaviria durante su participación en las II Jornadas de Urbanismo en la Costa Brava, organizadas por una comisión de la Diputación Provincial y celebradas en Sant Feliu de Guíxols en 1970. Por entonces responsable de Sociología del Instituto de Estudios de Administración Local (actualmente Instituto Nacional de Administración Pública), su intervención trató de alertar sobre el excesivo funcionalismo de las políticas públicas en materia de urbanismo. Rodeado de burócratas franquistas, Gaviria realizó sin tapujos un alegato en defensa del espacio urbano y la necesidad de ir más allá del interés especulativo de 
bloques y casas, con el fin de imaginar y construir un urbanismo turístico de calles y plazas que fundiera paisaje natural y obra humana (sic). Desde una posición fuertemente influenciada por el pensamiento del filósofo marxista Henri Lefebvre, sentenció: "Ahora sólo se piensa en el vacío como el espacio residual después de haber edificado los bloques. El promotor quiere construir muchas viviendas en muy pocos metros cuadrados [...] (Aconsejo a los municipios turísticos) que no consideren el espacio urbano como un mal inevitable e incurable, sino que lo trataran como algo artificial que puede completar e incluso superar a lo natural; que tuvieran un poco de imaginación al pensar en los objetos que componen la escena urbana" (Gaviria, 1970).

Toda esta situación de desenfreno urbanizador, llaman la atención también de algunos escritores de la zona. Turistes, sirenes $i$ gent del país, del ampurdanés Manuel Costa-Pau (1966) y Vint anys de turisme a la Costa Brava, del lloretense Esteve Fàbregas i Barri (1970) son dos obras de enorme valor producidas fuera de la academia, pero ricas en aportaciones antropológicas y sociológicas respecto al nacimiento y despliegue del desarrollo turístico en la Costa Brava. A diferencia del de Barbaza, estos dos textos son el relato perplejo de un mundo nuevo que se expande y lo trastoca todo, un mundo que ha venido para quedarse y ante el cual no cabe resistencia posible. La novela Les pianos mécaniques, del francés Henri-François Rey (1965) o la obra fotográfica de Xavier Miserachs reunida en el libro Costa Brava show (1966), son dos ejemplos más de la heterogeneidad de abordajes existente.

La hipótesis del impacto nacida en los 60, configura los primeros relatos académicos y su influencia se deja ver hasta nuestros días. ros días. Los diagnósticos de situación, como el trabajo firmado por Ernest Lluch i Pere Campistol (1972) sobre la economía, los precios del suelo y los recursos industriales en la Costa Brava, o las convocatorias de corte más crítico, como el Debat Costa Brava empiezan a labrar un terreno aún árido. El Debat Costa Brava, celebrado en 1976, se convierte en un acto de resistencia intelectual al paradigma urbanístico y territorial predominante. Este encuentro es el primero entre profesionales del sector turístico, académicos, organizaciones civiles y representantes de la administración pública, y servirá para tratar un buen número de controversias territoriales, económicas y sociales de la Costa Brava. Los objetivos del Debat son expresados por los mismos organizadores: "Fer-se ressó de la inquietud popular davant dels problemes actuals de la Costa Brava; analitzar la insatisfacció col-lectiva respecte al seu estat actual, estudiant les causes i processos que han conduit a aquesta situació; recollir tota mena d'aportacions que puguin servir de base per a un desenvolupament més racional i humà de la Costa Brava en el marc de les comarques gironines" (Aragó, 1996). A este encuentro no asisten ni hoteleros ni miembros de la Comisión de Información, Turismo y Educación (CITE). La crítica generalizada a los modelos de crecimiento urbano, turístico y económico, caen en saco roto pero permiten gestar un relato en defensa del territorio que a día de hoy sigue marcando las reivindicación y luchas de los movimientos sociales organizados de la Costa Brava.

Precisamente, unos de los académicos asistentes al Debat, el sociólogo catalán Jordi Estivill, será el próximo en publicar un artículo científico sobre el contexto turístico y social de la Costa Brava. Lloret de Mar: destruccions y resistències d'un poble en mans del turisme (1979) es el primer texto que, desde el marco de las llamadas teorías impactológicas, analiza las transformaciones provocadas por el auge de turismo salvaje -como lo define Estivill- en uno de los epicentros de la Costa Brava. El texto de Estivill sigue siendo excepción entre los temas que preocupan a la joven sociología y antropología estatal ${ }^{9}$. Sólo otro sociólogo, el ya mencionado Mario Gaviria, compartirá por entonces la atención hacia el turismo y su incidencia en las transformaciones sociales, urbanas y medioambientales.

Por fin, entrada la década de los 80, esta tendencia empieza a cambiar, y lo hace por todo lo alto. En el mismo 1980, el geógrafo británico Richard Butler pública The concept of a tourist area cycle of evolution: implications for management of resources, un artículo que marcará hasta la fecha un antes y un después en el análisis de los destinos turísticos. Y lo hará apoyándose precisamente en el ejemplo de la Costa Brava para ilustrar lo que este autor llamará "etapa de estancamiento" dentro de su teoría del Ciclo de Vida de los Destinos Turísticos, inspirada en cierto modo en el Irridex (índice de irritación) que Víctor Doxey desarrolló en 1975. La referencia de Butler es sin duda significativa y permite una primera ruptura con el optimismo crecentista. Con Butler se inaugura un período más fértil en investigaciones y publicaciones. Una figura destacada en este periodo ses Joan Cals, economista y profesor de la Universitat Autònoma de Barcelona (UAB).

Los primeros trabajos de Cals sobre la Costa Brava fueron escritos en la segunda mitad de los setenta. Con Les processus d'urbanisation touristique sur la Costa Brava (1976) y El sector turístic i la funció residencial de la Costa Brava (1978), este último formando parte además de las actas del Debat Costa Brava, Cals retoma el camino abierto por la mirada no complaciente de Barbaza. A partir de 1980, cuando completa su tesis de doctorado L'hoteleria tradicional en el context d'una política de turisme 
de masses, se sitúa como el máximo referente académico en el estudio de la Costa Brava. Ese trabajo le sirve de base para publicar dos años después el libro "La costa Brava i el turisme. Estudis sobre la política turística, el territori i l'hoteleria, donde analizará a fondo la transformación urbanística la Costa Brava desde la llegada del turismo, poniendo especial atención al desarrollo de urbanizaciones y al consumo individualizado del espacio que acarrean.

Aunque no podamos hablar de una obra voluminosa y sostenida en el tiempo, la importancia que sus enfoques y líneas de trabajo tienen sobre el resto de los investigadores interesados en la Costa Brava es notable. Tras Barbaza y Cals, la transformación del espacio y de la vida cotidiana en la Costa Brava se convierten en dos de los temas estrella. Algo que por otro lado no sorprende si se atiende al clima imperante durante las dos últimas décadas del siglo XX, llamadas a ser las de la reconversión turística. Se consuma la transformación definitiva de la base económica de los municipios turísticos de la Costa Brava, los cuales asumen un modelo de monoproducción de creciente especialización en el sector servicios y la construcción. El resultado inmediato es un crecimiento acelerado del espacio dedicado a los usos residenciales y a las actividades de consumo y ocio, en detrimento del dedicado a las actividades agrícolas y forestales, industriales y del turismo "tradicional"10 (Valdunciel, 2011).

Gerda K. Priestley, profesora de geografía en la Universitat Autònoma de Barcelona (UAB) y de origen norirlandés, trabaja también sobre el impacto que ese desarrollo urbanístico y turístico causa en el territorio y su fisionomía (Priestley, 1986). Otros investigadores, como el geógrafo estadounidense Philip C. Emmi y la economista catalana M. Àngels Santigosa, analizan las políticas de desarrollo urbano y uso de la tierra en su artículo Urban development, land use planning and political change: The case of Costa Brava, Spain (1989). Vinculados a esta producción, algunos investigadores abordan también la relación conflictiva del turismo y el territorio, como es el caso del alemán Konrad Tyrakowski (1986) o el madrileño Manuel Valenzuela (1986), este último vinculado al equipo de Joan Cals. Los geógrafos Arthur Morris y Gordon Dickinson, junto a Gerda K. Priestley, son otros que se suman al estudio de esta dimensión conflictiva del uso de la tierra (Morris et al., 1989). Respecto al trabajo de estos investigadores escoceses, merecen también atención los artículos Tourism and town planning in Catalonia", de Morris (1985) y Políticas de desarrollo turístico, entre el crecimiento y la conservación del territorio, de Morris y Dickinson (1987). La década de los 80 se completa con una nueva visita de Yvette Barbaza a la Costa Brava, al ser invitada en 1986 a las Jornades tècniques sobre turisme i Medi Ambient, en Sant de Guíxols. Su ponencia Consommation (production d'espace par le tourisme) da cuenta de cómo sigue atenta a los devenires de la producción turística del espacio veinte años después de la publicación de su tesis.

En 1984 el escritor londinense Norman Lewis publica la novela de tintes antropológicos Vices of the Old Sea. La obra está ambientada en esa Costa Brava en transición que años atrás dibujó Barbaza. Aunque la primera edición en lengua castellana no se edita hasta 1992, la historia de Lewis termina siendo un original prólogo en el tiempo a la etnografía que pública en 1989, el antropólogo catalán y profesor en la Universidad de Massachusetts Oriol Pi-Sunyer, como capítulo de una de las obras fundacionales de la antropología del turismo a nivel mundial, Host and Guests, the anthropology of tourism, coordinado por la antropóloga californiana Valene L. Smith (1989). En Changing perceptions of tourism and tourists in a Catalan resort town (1989), Pi-Sunyer analiza las representaciones que los habitantes generan hacia el turismo y los turistas en una población de la Costa Brava que el autor esconde bajo el nombre ficticio de Cap Lloc.

Dos años antes, la antropóloga británica Alison Lever publica en Annals of Tourism Research el articulo Spanish tourism migrants: the case of Lloret de Mar (1987). Encuadrado en los llamados estudios rurales, el texto de Lever traza el recorrido laboral y migratorio de los trabajadores andaluces en Lloret de Mar, prestando especial atención a la división sexual de dicha migración. Su trabajo ha tenido gran difusión y se ha situado por méritos propios como uno de los referentes en materia de turismo y trabajo.

Un breve punto y aparte. Como he ido señalando a lo largo de esta cronología descriptiva, existe una gran diversidad en lo que a referencias bibliográficas se refiere. Aquí solo estoy dando cuenta de algunas, principalmente de aquellas que considero más relevantes para establecer una primera cronología sobre ese objeto de estudio llamado Costa Brava. Dentro de esa selección, he creído conveniente reconocer también la tarea que a partir de finales de los ochenta lleva a cabo la Revista de Girona, la publicación que edita la Diputació de Girona desde 1955. Su contribución al estudio de la Costa Brava debe ser mencionado, no solo por la significancia de los temas tratados sino también por la persistencia y regularidad de sus publicaciones. A lo largo de todas estas décadas, ha ido publicando análisis de carácter histórico (Roig, 2014; Playà, 2012; Aragó, 2010, Turró, 2005; Pericot, 1959), social (Saló, 2004; Massaguer, 1997; Lanao et al., 1990; Pericot, 1960) o turístico (Perpinyà, 2009; Calabuig, 2004; 
Espinet, 2000; Fortià, 1997; Mundet, 1997). Els estrangers residents a la Costa Brava (Paunero, 1988) fue el primer artículo de una larga serie que llega, por el momento, hasta 2014. Para muchos de sus autores, la Revista de Girona ha sido un espacio privilegiado donde publicar investigaciones, ensayos, reflexiones o notas históricas sin los corsés ni las exigencias formales de las revistas científicas. Esto ha permitido difundir un valioso conocimiento entre un público que en el común de los casos está lejos de los formatos académicos.

Sigo. La década de los ochenta termina con un pronóstico territorial y social aún más pesimista que la anterior. Y la del noventa, arranca de forma similar a como empezó la de los setenta, con nuevos debates acerca del devenir de la Costa Brava. De alguna forma se da por terminado un periodo y por inaugurado el siguiente. Ejemplo de esto es el simposio El Paisatge de la Costa Brava: passat, present $i$ futur, organizado por el Grup Local de Defensa del Medi Ambient y celebrado en Lloret de Mar en diciembre de 1991. En este encuentro, vuelve a estar invitada Ivette Barbaza, quien defiende en su presentación iniciar una política de crecimiento cero como única vía posible para salvar el litoral. Sus palabras no son bien recibidas entre las autoridades locales y el sector empresarial; unos y otros defienden una política continuista y alertan del peligro de los planeamientos decrecentistas. De fondo está la hipótesis del estancamiento o de la fase de madurez, relato que dominará toda esa década. Tourism and local awareness: Costa Brava, nuevamente de Arthur Morris, entra de lleno en esta cuestión al analizar la actitud cambiante de ciertos grupos sociales a medida que se va desarrollando el turismo, y cómo estos actores contraponen una serie de valores ecológicos, históricos y culturales a las actuaciones de la industria turística sobre el territorio (Morris, 1996).

La falta de una universidad de cercana que haga de referencia no ayuda tampoco a fomentar una agenda sostenida de investigaciones en la Costa Brava. Esta situación no cambia hasta finales de la década de los noventa. Desde que se oficializa el traspaso de las competencias en educación a la Generalitat en 1980 y de los servicios en materia de turismo en 1982, tienen que pasar aún nueve años más para que se ponga en marcha el Institut Superior d'Estudis Turístics (INSETUR) y la Escola Oficial de Turisme (bajo el paraguas del primero). El siguiente movimiento es en 1998, cuando la Escola Oficial de Turisme pasa a ser un centro adscrito a la Universitat de Girona, normalizando así el decreto de dos años antes según el cual los estudios superiores de turismo debían desarrollarse en la universidad o en centros-escuelas adscritas a ella. En 2002 la UdG absorbe definitivamente la Escola Oficial de Turisme, que pasa a denominarse Escola Universitària de Turisme. Con la regularización de los estudios universitarios de turismo, en 2007 se crea la actual Facultat de Turisme de la UdG y el Institut Superior d'Estudis Turístics pasa a denominarse Institut de Recerca en Turisme, manteniendo el acrónimo INSETUR.

$\mathrm{El}$ asentamiento de este centro universitario es fundamental para ubicar el papel que empiezan a desempeñar los llamados estudios de gestión ${ }^{11}$. La maltrecha y masificada Costa Brava necesita encontrar soluciones a su agotamiento. Esto pasa por generar nuevas estrategias de comercialización y nuevos productos turísticos que generen valor y renueven el destino. El dogma crecentista ya no pasa solo por el desarrollo urbanístico (que por otro lado no se detiene), ahora las estrategias innovadoras de desconcentración, desestacionalización, diferenciación y segmentación son la punta de lanza donde convergen los poderes públicos y los agentes académicos -en el papel de consultores- en aras de mejorar la competitividad y el posicionamiento de los destinos.

Así a partir de los 90, y en lo que a la Costa Brava se refiere, la UdG deviene un centro de producción de estudios de gestión, asesoría y diseño de planes de actuación. Estos enfoques hacia lo management predominarán a lo largo de esa década y hasta el final de la primera del nuevo siglo. La llamada a la innovación del sector ante un mercado en transformación (Méndez, 1995), la puesta en marcha de nuevos productos culturales (Cànoves y Prat, 2011; Roca et al, 2009), las actuaciones y estrategias que llevan a cabo los destinos de la Costa Brava para readaptarse a esas nuevas tendencias (Sardà et al, 2005; Donaire et al, 1997), y en general la transición de un paradigma fordista a otro de posfordista (Mundet, 2000; Donaire, 1997) son los ejes principales de trabajo. Como excepción, cabe destacar el trabajo de tesis doctoral que Carolina Martí realiza sobre la transformación del paisaje ambiental y urbanístico de la Costa Brava a lo largo de los últimos 50 años (Martí, 2005), una obra que devuelve ciertos matices teòricos y metodológicos que por entonces parecen estar algo olvidados.

Como sucede con la UdG, el impulso institucional para la creación de centros e institutos de investigación y difusión del patrimonio catalán empieza a ser una realidad también a partir de mediados de los 90. El Centre de Promoció de la Cultura Popular i Tradicional Catalana (CPCPTC), el Inventari del Patrimoni Etnològic de Catalunya o el Observatori per a la Recerca Etnològica, todos vinculados y dependientes del Departament de Cultura de la Generalitat de Catalunya, son ejemplo de ello. Esto 
tendrá, por supuesto, una incidencia sobre el estudio de la Costa Brava. Por ejemplo la puesta en marcha del "Inventari del patrimoni etnològic maritimopesquer de la Costa Brava" a iniciativa de la Associació Cultural d'Amics del Cau de la Costa Brava-Museu de la Pesca y financiado por el CPCPTC. Este proyecto es llevado a cabo por el Grup d'Estudis Socials de la Pesca Marítima (perteneciente a la UdG) entre 1995 y 1999, bajo la coordinación del antropólogo Joan Lluís Alegre.

Con la entrada del nuevo siglo y siempre de la mano del Inventari del Patrimoni Etnològic de Catalunya (IPEC), se organizan dos citas importantes en el terreno etnográfico. En 2003 tiene lugar en Palamós la I Jornada d'Etnologia a la Costa Brava bajo el título de Recerca etnològica i interpretació del patrimoni en un entorn turístic. Cuatro años después, en 2007, se retoma la iniciativa y se organizan las II Jornada d'Etnologia a la Costa Brava, en esta ocasión con el título de La recerca en el camp de l'etnologia marítima i pesquera. En el momento de escribir este texto, se ha anunciado la celebración en octubre de 2019 de una (nueva) I Jornada de Recerca Etnològica a la Costa Brava, organizada en Torroella de Montgrí con el apoyo del Observatori del Patrimoni Etnològic i Immaterial (antes Observatori per a la Recerca Etnològica).

De nuevo fuera del ámbito específicamente etnológico, otra fecha a destacar es la celebración en Girona del II Debat Costa Brava (2005). Organizado esta vez por el Col-legi d'Arquitectes de Catalunya, su repercusión es menor que la de su antecesor pero merecen ser destacadas al menos tres presentaciones: la del profesor de geografía de la UdG José Antonio Donaire, titulada La lògica espacial del turisme a la Costa Brava (Donaire, 2005), la del también geógrafo Juli Valdunciel, que versa sobre el proceso de transformación urbana en el sur de la Costa Brava (Valdunciel, 2005), y la del catedrático de economía aplicada de la Universitat Autònoma de Barcelona (UAB) Josep Oliver, quien expone las causas del agotamiento del modelo turístico imperante y las alternativas de renovación o transición hacia uno nuevo en su ponencia El model turístic de la Costa Brava a l'inici del s. XXI: estació d'enllaç o final d'etapa? (Oliver, 2005).

Finalmente, y para dar cierre a esta cronología, me gustaría señalar algunas investigaciones que durante esta última década se han desviado del debate en torno a la renovación o transformación del destino "maduro", y han recuperado elementos de crítica al desarrollo turístico y urbano actual. Una ràpida muestra de ello, son los trabajos entorno a la degradación del modelo de ciudad mediterránea en favor de la ciudad dispersa (Valdunciel, 2011), el análisi de las debilidades de los marcos institucionales de los espacios marinos protegidos (De la Cruz et al, 2012), el diseño de políticas públicas más participativas y de consenso (Balagué y Navinés, 2013), la gestión y el control de un recurso tan fundamentales y crucial en la zona como es el agua (Pavón et al, 2018; Gabarda et al, 2016, 2015) o los impactos que supone el paso definitivo del mundo rural al urbano con la expansión de la industria del turismo, de la residencia vacacional y de las infraestructuras alrededor de los centros rurales tradicionales (Cuadrado et al, 2017). Así mismo, y recuperando también en parte la orientación crítica y etnográfica de aquellas primeras investigaciones de los años 80, cabe señalar el trabajo de la historiadora Mary Nash sobre dinámicas de neocolonialidad y masculinidades en la Costa Brava durante el tardofranquismo (Nash, 2017), la tesis en antropología social de Massimiliano Sassi sobre la relación entre las prácticas multilingües de la población residente en Empuriabrava y la exclusión laboral (Sassi, 2018), y finalmente la de uno mismo, también desde la antropología social, sobre las dinámicas sociales de apropiación turística del espacio en Lloret de Mar (Yanes, 2016). Estos últimos trabajos cierran, por el momento, más de medio siglo de investigaciones científicas sobre la Costa Brava

\section{Conclusiones}

Las limitaciones de esta revisión ya han sido expuestas al inicio, pero merece a pena insistir. Se han priorizado trabajos y formatos de acuerdo con un criterio que permitiera reunir la producción centrada específicamente en la Costa Brava, y se han discriminado aquellos otros que tocaran la cuestión de un modo más secundario o periférico. Esto ha hecho así con la intención de construir un eje central de esta literatura específica. Es tarea de otros investigadores interesados en el tema, tirar del hilo de las múltiples aristas que se desprenden.

Como conclusión y cierre, quisiera recuperar cuatro cuestiones que considero especialmente relevantes y señalar dos implicaciones también importantes a la luz de los resultados. En primer lugar, en el global de la bibliografía debe reconocerse el esfuerzo holístico realizado durante este tiempo. Esto ha permitido disponer de valiosos análisis sobre los múltiples fenómenos que configuran este territorio. Ahora bien, si estrechamos el foco, observamos también que, a lo largo de medio siglo, las disciplinas geográficas o 
de análisis económico y empresarial, han sido las protagonistas de estos análisis. Y si bien su agenda de investigación ha estado muy vinculada a los diversos procesos de transformación urbana/territorial y a los cambios de tendencia referidos al consumo, se echa en falta un mayor diálogo multidisciplinar, sobre todo con aquellos enfoques menos centrados en lo turístico y más en los contextos sociales donde el turismo es una realidad estructurante.

Siguiendo este argumento, los estudios críticos con el modelo de desarrollo territorial y turístico han sido predominantes hasta por lo menos el inicio de la década de los 90. Con mayor o menor intensidad, la mayor parte de las investigaciones y textos generados entre los 60 y 80 cuestionaron las políticas de gestión territorial, y sus planteamientos y análisis se enfrentaron al imperante sentido común del desarrollismo. Muchas de estas investigaciones estuvieron vinculadas a los Departamentos de Economía y de Geografía de la Universitat Autònoma de Barcelona (UAB), que además contaron con el interés de un número nada despreciable de investigadores extranjeros. A partir de la década de los 90 y ya con la Universitat de Girona (UdG) como centro académico de referencia, los estudios de gestión se abren paso. Es la época en la que consolida el marco teórico de la reconversión de los destinos maduros. Estos trabajos son la antesala de la colaboración que se afianza a partir del año 2000 entre el sector turístico público-privado y la universidad, siendo el diseño y la difusión de nuevos productos turísticos (turismo cultural, sostenible, patrimonial...) el punto de partida. Esta orientación hacia los estudios de planificación, márquetin, mercado y formación laboral se mantiene en buena medida hasta hoy.

En tercer lugar, merece también un reconocimiento explícito la labor colectiva que ha dado origen, a lo largo del territorio, a debates y jornadas sobre multitud de problemáticas asociadas al desarrollo y devenir de la Costa Brava. Impulsados por instituciones públicas, organizaciones civiles y movimientos sociales, estos encuentros han permitido hilar puentes con el mundo académico e incluso generar problematizaciones alternativas a las de sus programas de investigación. Su impacto e incidencia social ha sido importante, y con el paso de los años (y las décadas) siguen siendo un foco de resistencia intelectual que busca sus espacios de incidencia política.

Respecto a las implicaciones, deseo recalcar dos cuestiones íntimamente vinculadas. Por un lado, la identidad del objeto de estudio Costa Brava se construye y se transforma de acuerdo con contextos académicos cambiantes, pero también a disputas sociopolíticas (de tipo urbano) y representaciones mediáticas. Por otro, y atendiendo al contendido de los trabajos señalados, la Costa Brava es en sí misma una construcción social resultado de controversias que implican diferentes tipos de posiciones, saberes y prácticas. Desde el punto de vista antirrepresentacional que he defendido al inicio, no existe una Costa Brava "tal cual es", prelingüística o con cualidades inmanentes. El conocimiento entorno a la Costa Brava está marcado por los actores -científicos o no- que lo han producido desde hace más de un siglo. Las políticas turísticas, la literatura, el urbanismo o las ciencias sociales son prácticas sociales que construyen diferentes versiones -a veces complementarias o formando alianzas- del universo Costa Brava. Esto, lejos de ser vaguedad, debería alentar una ruptura con la lógica de la aplicación y abrir el objeto de estudio en toda su amplitud, dotándolo de profundidad y resaltando su mutabilidad. Desviándonos de la aplicabilidad mercadotécnica, estaríamos más cerca de retomar una agenda de investigación que pensara también la dependencia turística, el colapso ecológico, la explotación laboral o las múltiples discriminaciones encarnadas en la vida cotidiana de sus habitantes.

\section{Bibliografía}

Aragó, N. J. 2010. "La Costa Brava abans dels turistes", en Revista de Girona, 263. 128-129.

Aragó, N. J. 1996. "El Debat Costa Brava, pas a pas”, en Revista de Girona, 179. 62-70.

Balagué, J., Navinés, F. 2013. "Sistema de indicadores para la gestión sostenible de un destino turístico: aplicación a la Costa Brava centro", en Harvard Deusto Business Research, 1(2). 132-146.

Ballard, K. G. 1958. Five roads to S'Agaró. Londres: Boardman \& Co.

Ballester, I. 1966. "La Costa Brava. Un modelo de estudio comarcal", en Revista de Estudios de la Administración Local. Madrid.

Barbaza, Y. 1986. "Consommation (production d'espace par le tourisme)", en Jornades tècniques sobre turisme i Medi Ambient. Sant Feliu de Guíxols. 45-86.

Barbaza, Y. 1966 [1988]. El paisatge humà de la Costa Brava. Edicions 62: Barcelona.

Butler, R. W. 1980. "The concept of a tourist area cycle of evolution: implications for management of resources", en Canadian Geographer, XXIV (1). 5-12. 
Calabuig, S. 2004. La competència entre els municipis de la Costa Brava: cooperar i competir", en Revista de Girona, 224. 30-35.

Cals, J. 1987. L'Alt Empordà. Recursos i Estructura Econòmica. Barcelona: Caixa d'Estalvis de Catalunya.

Cals, J. 1982. La costa Brava i el turisme. Estudis sobre la política turística, el territori i l'hoteleria. Barcelona: Kapel.

Cals, J. 1980. L'hoteleria tradicional en el context d'una política de turisme de mases. Tesis doctoral. Departament de Ciències Econòmiques. Universitat Autònoma de Barcelona.

Cals, J., Turró, J. 1978. "El sector turístic i la funció residencial de la Costa Brava”, en Debat Costa Brava. Cambra de Comerç i Indústria de Girona.

Cals, J., Esteban, J., Teixidor Felip, C. 1977. "Les processus d'urbanisation touristique sur la Costa Brava”, en Revue géographique des Pyrénées et du Sud-Ouest, vol. 48 (2). 199-208.

Calvo, Ll. 1997. Historia de la antropología en Cataluña. Madrid: CSIC.

Cànoves, G., Prat, J. M. 2011. "La Costa Brava en Catalunya: del tradicional modelo de sol y playa a las nuevas opciones de turismo cultural”, en J. Fernando Vera (ed.) Seminario Internacional Renovación y Reestructuración de Destinos Turísticos Consolidados del Litoral. Alicante: Instituto Universitario de Investigaciones Turísticas, Universidad de Alicante.

Centre Excursionista de Catalunya. 1932. La Costa Brava. Barcelona: CEC.

Ciuró, J. 2011. La Costa Brava de los años sesenta. Lloret de Mar: Club Marina Casinet.

Clyne, D. 1959. Your guide to la Costa Brava. Londres: Alvin Redman.

Colomer, L. 1930. Petita Guia de la Costa Brava i del Baix Empordà. Palamós Sant Joan de Palamós, Calonge, Sant Antoni i la Fosca. Edición a cargo del autor.

Costa-Pau, M. 1966. Turistes, sirenes i gent del pais. Ariel: Barcelona.

Cuadrado, S., Durà, A., \& Salvati, L. 2017. "Not only tourism: unravelling suburbanization, second-home expansion and "rural" sprawl in Catalonia, Spain", en Urban Geography, 38:. 66-89

De la Cruz, R., Vendrell, B., \& Pascual, J. 2012. "¿Quién rompe las normas? Dilemas e ineficacias en la gestión turística de los espacios marinos protegidos”, en Santana, A., Rodríguez, A. J., Díaz, P. (coord.) Responsabilidad y turismo. Colección Pasos, 10.

Donaire, J. A. 2005. "La lògica espacial del turisme a la Costa Brava", en Debat Costa Brava. Girona: COAC.

Donaire, J. A. 1997. El turismo a los ojos del postmodernismo. Una lectura desde la dialéctica socioespacial: la Costa Brava, Tunicia y los malls. Tesis Doctoral. Departament de Geografia. Universitat Autònoma de Barcelona.

Donaire, J. A., Fraguell, R. M., Mundet, Ll. 1997. "La Costa Brava ante los nuevos retos del turismo", en Estudios Turísticos, 133. 77-96.

Emmi, P. C., Santigosa, M. A. 1989. "Urban development, land use planning and political change: The case of Costa Brava, Spain", en Land Use Policy, Vol. 6(2). 103-120.

Espinet, J. M. 2000. "La Costa Brava, una zona competitiva en preus", en Revista de Girona, 203. 36-41.

Estivill, J. 1979. "Lloret de Mar: Destruccions i resistències d'un poble en mans del turisme", en: Papers: Revista de sociologia. Vol. 10. Universitat Autònoma de Barcelona. 175-201.

Fàbregas, E. 1970. Vint anys de turisme a la Costa Brava. Selecta: Barcelona.

Fidrmuc, P. 1948. Una piragua en la Costa Brava. Barcelona: Iberia.

Fortià, R. 1997. "L'ordenació de la Costa Brava i la preservació de l'entorn natural", en Revista de Girona,180. 26-43.

Gabarda, A., Fraguell, R.M., Pavón, D., \& Ribas, A. 2016. "Tourist development and wastewater treatment in the Spanish Mediterranean coast: The Costa Brava case study", en International Journal of Sustainable Development and Planning, 11. 245-254.

Gabarda, A., Ribas, A., \& Daunis-i-Estadella, J. 2015. "Desarrollo turístico y gestión eficiente del agua. Una oportunidad para el turismo sostenible en la Costa Brava (Girona)", en Investigaciones Turísticas, 9. 50-69.

Gaviria, M. 1970. II Jornadas de Urbanismo de la Costa Brava. Desconocido. [Recuperado de https:// core.ac.uk/download/pdf/39067682.pdf]

Lanao, P., Vinyoles, \& C., Torns, M. 1990. "La Costa Brava, entre l'esperança i la desesperació", en Revista de Girona, 139. 24-31.

Lever, A. 1987. "Spanish tourism migrants: the case of Lloret de Mar", en Annals of Tourism Research, vol. 14 (4). 449-470.

Lewis, N. 1984. Voices of the Old Sea. Boston: Da Capo Press.

Lluch, E., Campistol, P. 1972. Les conseqüències comarcals del turisme a la Costa Brava (de Sant Feliu de Guíxols a Palamós). Barcelona: Caixa de Catalunya. 
Luján, N. 1958. La Costa Brava. Barcelona: Noguer.

Marín, M. 2001. "Historiadores locales e historiadores universitarios. La historiografía española en su contexto internacional, 1948-1965”, en Forcadell, C.; Peiró, I. (coords). Lecturas de la historia. Nueve reflexiones sobre Historia de la Historiografía. Zaragoza: Institución «Fernando el Católico». 97-149.

Martí, C. 2005. La transformació del paisatge litoral de la Costa Brava: Anàlisi de l'evolució (1956-2003), diagnosi de l'estat actual i prognosi de futur. Tesis doctoral. Institut de Medi Ambient. Universitat de Girona.

Massaguer, S. 1997. "La vida col.lectiva a la Costa Brava i els aspectes humans", en Revista de Girona, 180. 50-53.

Méndez, R. 1995. Economía del sector turístico. Una aplicación de la demanda turística en la Costa Brava. Tesis doctoral. Facultat d'Economia i Empresa. Universitat de Barcelona.

Miserachs, X. 1966. Costa Brava show. Barcelona: Kairós.

Morris, A. 1996. "Tourism and local awareness: Costa Brava, Spain", en Priestley, G. K., Arwel, J., Coccossis, H. (eds.) Sustainable tourism?: European experiences. Oxford University Press. 70-85.

Morris, A. 1985. "Tourism and town planning in Catalonia", en Planning Outlook, 28(2). 77-82.

Morris, A., Dickinson, G. 1987. "Tourist development in Spain: growth versus conservation on the Costa Brava", en Geography, 72(1). 16-25.

Morris, A., Dickinson, G., \& Priestley, G. 1989. "Land use change and conflict in the Bay of Roses, Costa Brava”, en Occasional Papers, 25. Glasgow: Department of Geography \& Topographic Science, University of Glasgow.

Mundet, Ll. 2000. "La Costa Brava y l'Estartit. Un ejemplo de transición entre distintos modelos turísticos", en Estudios y Perspectivas en Turismo, 9. 229-250

Mundet, Ll. 1997. "La situació económica i turística de la Costa Brava", en Revista de Girona,180. 44-49.

Nash. M. 2017. "Turismo y la Costa Brava: discursos neo-coloniales y de resistencia en los 1960s", en Chamouleau, B. (ed.) De colonialidad. Perspectivas sobre sujetos y género en la historia contemporánea de España. Madrid: Postmetrópolis

Oliver, J. 2005. "El model turístic de la Costa Brava a l'inici del s. XXI: estació d'enllaç o final d'etapa?", en Debat Costa Brava. Girona: COAC. 246-277

Parsons, J. J. 1973. "Southward to the sun: The impact of mass tourism on the coast of Spain", en Yearbook of the Association of Pacific Coast Geographers. Vol. 35. 129-146.

Paunero, F. X. 1988. "Els estrangers residents a la Costa Brava", en Revista de Girona, 131. 76-82.

Pavón, D., Gabarda-Mallorquí, A. \& Ribas, A. 2018. "What governance? The role of public and private stakeholders in water supply management in Mediterranean coastal tourist destinations: The case of the Costa Brava", en Water, 10(12). 1758.

Pericot, J. 1960. "Arquitectura de la Costa Brava", en Revista de Girona, 11. 19-24.

Pericot, Ll. 1959. "La primera descripción de la Costa Brava", en Revista de Girona, 6. 41-44.

Perpinyà, M. 2009. "Costa Brava segle XXI”, més enllà del sol i platja”, en Revista de Girona, 257. 54-55.

Pi-Sunyer, O. 1989. "Changing perceptions of tourism and tourists in a Catalan resort town", en Smith, V. L. (ed.) Hosts and guests. The anthropology of tourism. University of Pennsylvania Press. 187-199.

Playà, J. 2012. "El primer viatge «oficial» a la Costa Brava", en Revista de Girona, 274. 62-64.

Priestley, G. 1986. "El turisme i la transformació del territori: un estudi de Tossa, Lloret de Mar i Blanes a través de la fotografia aèria, 1956-1981", en Jornades Tècniques sobre turisme i Medi Ambient. Sant Feliu de Guíxols. 87-106.

Priestley, G., Mundet, Ll. 1989. "The post-stagnation phase of the resort cycle", en Annals of Tourism Research, 25(1), 85-111.

Quintana Trias, Ll. 2004. "Els turistes són els altres. Notes sobre el descobriment de la Costa Brava en la literatura catalana", en Rassegna Iberistica, 79. 49-65.

Quintana Trias, Ll. 2003. "Primitivisme i primarietat: la imatge de la Costa Brava en alguns autors catalans dels anys 20", en Professor Joaquim Molas. Memòria, escriptura, història. Publicacions de la Universitat de Barcelona. 845-59.

Roca, E., Villares, M., \& Ortego, M. I. 2009. "Assessing public perceptions on beach quality according to beach users' profile: A case study in the Costa Brava (Spain)", en Tourism Management, 30 (4). 598-607

Roca, F. 2000. Teories de Catalunya. Guia de la societat contemporània. Barcelona: Pòrtic.

Roig, S. 2014. "La mirada anglesa. Escriptors britànics a la Costa Brava", en Revista de Girona, 283. 76-77.

Saló, A. 2004. "Les segones residències. Anàlisi específica per a la Costa Brava", en Revista de Girona, 225. 30-35.

Sardà, R.; Mora, J.; Avila, C. 2005. "Tourism development in the Costa Brava (Girona, Spain) — how integrated coastal zone management may rejuvenate its lifecycle", en Vermaat, J.; Salomons, W.; Bouwer, L.; Turner, K. (eds). Managing European Coasts. Berlin: Springer. 291-314. 
Sassi, M. 2018. Mobility, work, and language hierarchy in a tourist community on the Costa Brava. Language and Intercultural Communication, 18:4. 377-389.

Solé de Sojo, V. 1929. La Costa Brava. Barcelona: Libreria Francisco Puig.

Torrent, J. 1958. La Costa Brava. Vista pels escriptors catalans. Barcelona: Barcino.

Tyrakowski, K. 1986. "The role of tourism in land utilization conflicts on the Spanish Mediterranean coast", en GeoJournal, 13(1). 19-26.

Turró, J. 2005. "Planificació i promoció turística a la Costa Brava dels anys 30", en Revista de Girona, 230. 39-45.

Valdunciel, J. 2011. Paisatge i models urbans contemporanis. Les comarques gironines (1979-2006): del desarrollismo a la globalització. Tesis Doctoral. Departament de Geografia, Història i Història de l'Art. Universitat de Girona. Girona.

Valdunciel, J. 2005. "Costa Brava Sud (1985-2001). La dinàmica accelerada de les transformacions en la realitat urbana", en Debat Costa Brava. Girona: COAC.

Valenzuela, M. 1986. "La residencia secundaria como factor de transformación y reordenación del sistema de asentamientos. Conflictos territoriales y retos urbanísticos", en Jornades Tècniques sobre turisme i Medi Ambient. Sant Feliu de Guixols. 107-128.

Vega, A. 2013. "Turismo, arte y mascarada en España: el caso de la Costa Brava”, en Anales de Historia del Arte, 23. 51-65.

VV.AA. 2010. La Costa Brava abans de la Costa Brava. Fotografies de la Casa de la Postal 1915-1935. Ajuntament de Roses.

Yanes, S. 2016. Abierto por vacaciones. Una etnografía de la apropiación turística en Lloret de Mar. Tesis doctoral. Departament de Antropologia Cultural i Història d'Amèrica i d'Àfrica. Universitat de Barcelona.

\section{Notes}

1 Señalo lo de controversias por el debate que aún hoy existe sobre el origen de la denominación Costa Brava. Autores como Josep Pla, Agustí Calvet (Gaziel), Vicenç Coma o Esteve Fàbregas defendieron en su día otras autorías y otros lugares de bautizo. En cualquier caso, en lo que unos y otros coinciden, es que la gestación de la marca Costa Brava nace ligada a los prohombres de la Lliga Regionalista.

2 Exponente político del catalanismo conservador. Su hegemonía se extiende a lo largo del primer tercio del siglo XX. Francesc Cambó fue la figura que lideró el partido hasta su desaparición en 1936. Tras el golpe de estado franquista, una parte importante de sus miembros se alinearon con el nuevo gobierno.

3 Recordemos que el nombre Côte d'Azur fue también ideado como marca turística, en este caso en 1887 y por el político y escritor francés Stéphen Liégeard.

4 "Es la indústria de los forasteros, de la que viven, no algunos pueblos, sino naciones enteras, como Suiza" (TdA).

5 Traducida y editada por la editorial Tusquets en 2013 bajo el título de Un hotel en la Costa Brava.

$6 \quad$ Notes disperses (Destino, 1969) o Cinco historias de mar (Destino, 1987) son dos ejemplos más. De hecho, hace pocos años David Viñas publicó Josep Pla i l'invent "Costa Brava”(A Contra Vent, 2013), una obra que se presenta como un viaje por la Costa Brava a través de las letras y la memoria de Josep Pla.

7 Ese mismo año Ignasi Ballester pública el artículo La Costa Brava. Un modelo de estudio comarcal, aunque es evidente que su alcance no es en absoluto comparable con la obra de Barbaza.

8 Un ejemplo de ello es la creación en 1970 del organismo político-empresarial Comunidad Turística de la Costa Brava, que tiene como objetivo principal la coordinación de los agentes económicos y políticos para facilitar el marco normativo y legal del fomento urbanístico, turístico y empresarial.

9 Recordemos que el trabajo doctoral de Ivette Barbaza fue realizado también en una universidad francesa. De hecho, el texto de Estivill, se basó en la investigación Saint Cyprien et Lloret de Mar: tourisme planifié et tourisme sauvage, que el sociólogo catalán realizó en la Universitat de Perpinyà, también en Francia.

10 Las comillas son del original

11 Me refiero a los "estudios de gestión" en turismo, como aquellos dirigidos a analizar o desarrollar estrategias de administración o creación de iniciativas o proyectos turísticos, sean estos públicos, privados o de diverso alcance y temática. Entrarían dentro del ámbito de las ciencias sociales aplicadas y se traducirían en la mayoría de casos en trabajos de asesoría o consultoría para el sector turístico. Dada esta orientación, normalmente establecen una relación su objeto de estudio neutral y con vocación tecnicista.

Recibido:

$13 / 11 / 2018$

Reenviado:

$19 / 04 / 2020$

Aceptado:

$20 / 05 / 2020$

Sometido a evaluación por pares anónimos 